\title{
On the theory of metal nanoparticles based on quantum mechanical calculation
}

\author{
Elias Saion* and Elham Gharibshahi \\ Department of Physics, Universiti Putra Malaysia, 34300 UPM Serdang, Selangor, Malaysia \\ Received 2 November 2010, Revised 1 December 2010, Accepted 25 December 2010, Available online 20 Januari 2011
}

\begin{abstract}
Metal nanoparticles have attracted considerable attention owing to their unusual physical and chemical properties from those of their molecular and bulk counterparts and are fundamental to surface science applications such as catalysts, optics, photonics, sensors, and spectroscopy. Traditionally, the optical absorption spectra are derived from the collective oscillations of free electrons of conduction band in metal nanoparticles as a consequence of incident electromagnetic radiation polarizing the nanoparticles. This phenomenon, known as the localized surface plasmon resonance, is unique to metallic nanostructures and has been modelled by Gustav Mei in 1908 based on the Maxwell's equations. It is the most-cited scientific paper of $20^{\text {th }}$ century and this classical approach is still used widely. However, the theory cannot account for quantum confinement effects of the electronic structure, the fundamental physical properties of metal nanoparticles. More satisfying treatment of photons interacting with metal nanoparticles is by a quantum theory approach. When UV-visible light impinging on a metal nanoparticle, occupied ground-state conduction electrons absorb photons and excite to higher unoccupied higher energy-state of the conduction band of the particle. In this development we used time-independent Schrodinger equation of the ground-state energy of Thomas-Fermi-Dirac-Weizsacker atomic model and also the density function in the final Euler-Lagrange equation was algebraically substituted with the absorption function. The total energy functional was computed numerically for isolated silver and gold nanospheres at various sizes. The electronic transitions within the conduction band are limited only by the Lagrange multiplier and the quantum number selection rules. The calculated absorption peaks fall within the experimental regimes. The results show a red-shift absorption peak increases with the increase of particle diameter corresponds to a decrease in the conduction band energy of metal nanoparticles.
\end{abstract}

| Metal Nanoparticles | Optical Absorption | Quantum Mechanical Calculation | Density Functional Theory |

® 2011 Ibnu Sina Institute. All rights reserved. http://dx.doi.org/10.11113/mjfas.v7n1.202

\section{INTRODUCTION}

The last two decades have witnessed a significant rapid growth in the study of metallic nanostructures. They have attracted much attention because of their surface effect (large surface-to-volume ratio) and quantum confinement effects (size-dependent properties). These affect their chemical and physical properties such as the catalytic, optical, electrical, and magnetic characteristics, which are distinctly different from their molecular and bulk counterparts. Noble metal nanoparticles, in particular Ag and $\mathrm{Au}$, exhibit a strong optical absorption of electromagnetic radiation in the UV-vis-NIR region [1,2]. This phenomenon has been a challenge in the field of nanoscience for the last one hundred years. According to the classical electrodynamics theory, the absorption is derived from the collective oscillations of free conduction electrons in a metallic nanostructure as a consequence of incident electromagnetic radiation polarizing the nanoparticle. These oscillations, known as the localized surface plasmon resonance (LSPR), are unique to metallic nanostructures and their resonance frequency is dependent on the nanoparticle characteristics such as the size, shape,

Corresponding author at: Department of Physics,

Universiti Putra Malaysia, 34300 UPM Serdang, Selangor, Malaysia

E-mail addresses: elias@science.upm.edu.my (Elias Saion) and chemical composition [3,4] and on the surrounding medium's properties [5]. Understanding the interaction between electromagnetic radiation and conduction electrons is vital in the fabrication of metal nanoparticles to control their electronic and optical characteristics. In recent years, the LSPR induced in Ag and Au nanoparticles has shown extensive applications in various fields such as catalysis [6], optics [7], optoelectronics [8], spectroscopy [9], biomedical applications [10], and electrochemical sensors [11].

Simulations of the properties of metal nanoparticles are equally important as the fabrication and characterization of these materials confined to small dimensions. There are a variety of different theoretical models to describe the optical absorption of metal nanoparticles [12-14]. Among the classical electrodynamics models, the Mie's theory [12], a solution based on the Maxwell's equations, provides excellent LSPR description for a spherical metal nanoparticle embedded in an optically dielectric matrix for a particle size very much smaller than the wavelength of light. The Gans theory [15], an extension of Mie's theory, is applicable to prolate or oblate particles averaged over all orientations within the dipole approximation. Alternatively, the optical properties of nanoparticles may be described by the Maxwell-Garnett theory [16] in terms of the effective complex dielectric functions of the particles and surrounding medium developed from the atomic 
polarizability and material density. The theory also includes the geometry and spatial distribution of the particles with two resonance modes coming from randomly oriented prolate nanoparticles. Nevertheless, the electrodynamicsbased theory cannot account for the energy discretization of conduction electrons, which is the fundamental electronic property of metal nanoparticles. The classical theory allows conduction electrons to oscillate at the wavelength of 200 $\mathrm{nm}$ or more in the confinement nanoparticles size of less than $20 \mathrm{~nm}$, which physically is difficult to imagine.

Considerable efforts have been made to calculate optical excitation and photoabsorption properties in metal nanoparticles of many-electron systems based on the timedependent density functional theory (DFT) [17-20]. Optical excitation and absorption of metal nanoparticles can basically be calculated using time-independent DFT, a ground state theory that requires the ground state conditions of conduction electrons and their properties [19]. This suggests that the optical absorption spectra of metal nanoparticles can be determined from the ground-state density through the Hamiltonion operator because it characterizes all of the energy states of a system. However, so far the direct energy states of conduction electrons of metal nanoparticles have not been seen in UV-vis absorption measurements. Instead, the absorption spectra of metal nanoparticles are often displayed as a broad spectrum originating from conduction electrons with exceptionally degenerate states. This is in marked contrast with excitation properties of metal nanoparticles such as luminescence [21] and fluorescence [22], where the quantized states are readily observed due to well-defined energy gaps between two occupied energy states. In the conduction band of metal nanoparticles, the electron-hole interaction is screened off, and conduction electrons behave as nearly free.

The present paper describes a fully quantum mechanical calculation of the absorption spectra of Ag and Au nanoparticles based on time-independent DFT. The particle is an isolated single solid metal sphere containing $N$ atoms arranged in a face-cantered cubic lattice structure. The total energy functional is the ground-state energy functional of the Thomas-Fermi-Dirac-Weizsacker atomic model to allow for the ground-state electron density to be finite at the lowest energy state of the conduction band. The optical absorption of conduction electrons may be calculated by DFT because there is a relationship between the electron density and the absorption. In this development, the electronic density function in the final Euler equation was algebraically substituted with the absorption. Our study includes calculations of Lagrange multipliers, lattice constants, electronic transitions, transition potentials, photon wave vectors, optical absorption spectra, and the number of atoms in $\mathrm{Ag}$ and $\mathrm{Au}$ nanospheres at different particle diameters.

\section{THEORY OF METAL NANOPARTICLES}

In quantum mechanical calculation, HohenbergKohn-Sham DFT [23-25] has been most widely used to study the electronic structures of many-electron systems such as nanostructures. The early foundations of DFT are due to the Hohenberg and Kohn theorem [24] and KohnSham equations [25], where the ground state electron density $\rho(\boldsymbol{r})$ is the basic variable, from which all ground state properties could be derived. For optical absorption of metal nanoparticles, the ground-state energy functional $E[\rho]$ may be taken from the Thomas-Fermi-DiracWeizsacker atomic model [23, 26-28], written as

$E[\rho(\boldsymbol{r})]=T_{T F}[\rho(\boldsymbol{r})]+\lambda T_{W}[\boldsymbol{r}]+\int \rho(\boldsymbol{r}) v(\boldsymbol{r}) d \boldsymbol{r}+V_{e e}[\rho]$

The first term, $T_{T F}[\rho(\boldsymbol{r})]$, is the kinetic energy of the Thomas-Fermi (TF) model in its original formulation of a local density approximation and expressed as a function of the electron density $\rho(\boldsymbol{r})$ of an infinite number of homogenous free electron gas systems at a given coordinate $\boldsymbol{r}$, given by

$T_{T F}[\rho(\boldsymbol{r})]=C_{k} \int \rho(\boldsymbol{r})^{5 / 3} d \boldsymbol{r}$

where $\quad C_{k}=\frac{3}{10} \frac{\hbar}{m}\left(3 \pi^{2}\right)^{2 / 3}$, the second term, $\lambda T_{W}[\rho(\boldsymbol{r})]$ is the von Weizsacker correction to the kinetic energy of the TF model by inclusion exchange and a correlation energy terms [28] for the inhomogeneity of the electron density as a gradient correction about the uniform gas. This is the correct kinetic energy functional for metal nanoparticles where the conduction electrons resemble a one-electron or a two-electron Hartee-Fock atom, given by

$T_{W}[\rho(\boldsymbol{r})]=\frac{1}{8} \frac{\hbar^{2}}{m} \int \frac{|\nabla \rho(\boldsymbol{r})|^{2}}{\rho(\boldsymbol{r})} d \boldsymbol{r}$

The parameter $\lambda$ may be obtained by some empirical arguments for the ground state energy [29-31]. The third term is the potential energy of the system, and the fourth term, expressed as $V_{e e}[\rho]=J[\rho]-K_{T F D}[\rho]$, is the potential energy functional for the effective electron-electron repulsion. $J[\rho]$ is the classical Coulomb energy of electron-electron interactions, and $K_{T F D}[\rho]$ is the ThomasFermi-Dirac (TFD) model, which refers to the non-classical exchange-correlation energy of a homogenous free electron gas system defined as containing all remaining quantum effects not captured by $J$ and kinetic energies $T$.

$J\left[\rho\left(\boldsymbol{r}, \boldsymbol{r}^{\prime}\right)\right]=\frac{1}{2} \iint \frac{\rho(\boldsymbol{r}) \rho\left(\boldsymbol{r}^{\prime}\right)}{\left|\boldsymbol{r}-\boldsymbol{r}^{\prime}\right|} d \boldsymbol{r} d \boldsymbol{r}^{\prime}$

and

$K_{T F D}[\rho(\boldsymbol{r})]=C_{e} \int \rho(\boldsymbol{r})^{4 / 3} d \boldsymbol{r} ; C_{e}=e^{2} \frac{3}{4}\left(\frac{3}{\pi}\right)^{1 / 3}$ 
By taking atomic units $\hbar=m=e=c=1$ throughout, the total energy functional, in the differential form to the second order is

$$
\begin{aligned}
& E[\rho]=C_{k} \int \rho(\boldsymbol{r})^{5 / 3} d \boldsymbol{r}+\frac{\lambda}{8} \int \frac{\mid \nabla \rho(\boldsymbol{r})^{2}}{\rho(\boldsymbol{r})} d \boldsymbol{r}+\int \rho(\boldsymbol{r}) \nu(\boldsymbol{r}) d \boldsymbol{r}+ \\
& \frac{1}{2} \iint \frac{\rho(\boldsymbol{r}) \rho\left(\boldsymbol{r}^{\prime}\right)}{\left|\boldsymbol{r}-\boldsymbol{r}^{\prime}\right|} d \boldsymbol{r} d \boldsymbol{r}^{\prime}-C_{e} \int \rho(\boldsymbol{r})^{4 / 3} d \boldsymbol{r}
\end{aligned}
$$

The exact ground state energy of the metal nanoparticles is the global minimum value of $E[\rho]$, and the density $\rho(\boldsymbol{r})$ that minimizes $E[\rho]$ is the exact ground state density $\rho_{0}$, namely

$E_{0}=E\left[\rho_{0}\right]=\min \left\{E[\rho(\boldsymbol{r})]: \rho \geq 0, \int \rho(\boldsymbol{r}) d \boldsymbol{r}=N\right\}$

where $E_{0}$ is the exact ground state energy, $E\left[\rho_{0}\right]$ is the minimized energy functional [24] and $N$ is the number of electrons in the conduction band. The ground-state electron density must satisfy the variational principle,

$$
\delta\left\{E[\rho]-\mu\left[\int \rho(\boldsymbol{r}) d \boldsymbol{r}-N\right]\right\}=0
$$

where $\mu$ is the Lagrange multiplier associated with the normalized density functional. For completely degenerate conduction electrons at absolute zero temperature, $\mu$ is the Fermi energy. This yields the Euler equation and is written as

$$
\mu=\frac{\delta E[\rho(\boldsymbol{r})]}{\delta \rho(\boldsymbol{r})}=v(\boldsymbol{r})+\frac{\delta T_{T F}}{\delta \rho(\boldsymbol{r})}+\lambda \frac{\delta T_{W}}{\delta \rho(\boldsymbol{r})}+\frac{\delta V_{e e}}{\delta \rho(\boldsymbol{r})}
$$

The Euler equation (9) may be presented in terms of functional derivatives,

$$
\begin{aligned}
& \frac{5}{3} C_{k} \int \rho(\boldsymbol{r})^{2 / 3} d \boldsymbol{r}+\frac{\lambda}{8}\left[\frac{|\nabla \rho(\boldsymbol{r})|^{2}}{\rho^{2}(\boldsymbol{r})}-2 \frac{\nabla^{2} \rho(\boldsymbol{r})}{\rho(\boldsymbol{r})}\right]+v(\boldsymbol{r})+ \\
& e^{2} \int \frac{\rho(\boldsymbol{r})}{\left|\boldsymbol{r}-\boldsymbol{r}^{\prime}\right|} d \boldsymbol{r}-\frac{4}{3} C_{e} \int \rho(\boldsymbol{r})^{1 / 3} d \boldsymbol{r}=\mu
\end{aligned}
$$

where, $r$, the displacement coordinate of the conduction electrons from the centre of nanosphere and is dependent on the Bohr radius $a_{0}$, atomic number $Z$, the principle quantum number $n$, the angular quantum number $l$, and the spin quantum number $s$. For completely ground-state conduction electrons at absolute zero temperature, $\mu$ is the Fermi energy. We found that the density of conduction electrons $\rho(\boldsymbol{r})$ of an atom is a function of atomic number $Z$ and absorption $\sigma(\boldsymbol{r})$. Since both $\rho(\boldsymbol{r})$ and $\sigma(\boldsymbol{r})$ are continuous functions, the transformation of the density functional energy $E[\rho]$ to the absorption functional energy $E[\sigma]$ can be made by algebraically substituting the electron density function with the absorption function in the EulerLagrangian equation (10). The final Euler-Lagrangian equation is the second order differential equation in terms of the absorption function and it may be solved numerically.

\section{NUMERICAL SIMULATION}

The absorption functional energy $E[\sigma]$ of the conduction electrons is dependent on various factors such as the atomic number, atomic mass, lattice constant, electron potential, ground-state local wave vector, number of atoms that make up a spherical volume, and quantum number selection rules $\Delta n \geq 1$ for principle quantum number and $\Delta l=0,1$ for angular quantum number. The conduction electrons receive a plane electromagnetic wave $x$ from photons and must overcome the ground-state electromagnetic wave vector $k_{n}$ of the conduction electrons to promote to the higher energy states. $k_{n}$ is directly proportional to the principle quantum number $n$ and inversely proportional with the number of atoms $N$ or $k_{n}=2 \pi n / N a$, where $a$ is the lattice constant. The Ag and $\mathrm{Au}$ nanoparticles are arranged in face-centred cubic structures with their lattice constant $a$ equal to 0.408 and $0.407 \mathrm{~nm}$, respectively. The ground-state energy of the conduction electrons has the principle quantum number $n=$ 5 and 6 for Ag and Au nanoparticles respectively. The Fermi levels $\mu$ were calculated for Ag and Au to be 5.49 and $5.53 \mathrm{eV}$, respectively. By using Bloch's theorem of $\sigma(x)=\sigma(x) \exp (j k N a)$ and the Born-von Karman condition [32], we reach the boundary conditions at the end points of the nanocrystal of $\sigma_{0}=0$ and $\sigma_{N}=0$, where the two end points overlapped to form a lattice loop.

For numerical calculation, the absorption and wavelength in the final Euler-Lagrangian of second order differential equation are discretized into $\sigma_{i}$ and $x_{i}$, by $\partial^{2} \sigma(x) / \partial x^{2}=\left(\sigma_{i+1}+\sigma_{i-1}-2 \sigma_{i}\right) / \Delta^{2} \quad$ and $\partial \sigma(x) / \partial x=\left(\sigma_{i+1}-\sigma_{i-1}\right) / 2 \Delta$, where $i=0,1,2, \cdots \cdots N$ are integers representing the number of atoms that made up the spherical volume of a given diameter [33]. The multivariate equations may be solved by a trapezoid integration method using the Newton iterative program with a mesh size $\Delta=0.01$. Numerical simulations were carried out on single particles of different sizes. Thus, the ground state energy of the conduction electrons is dependent on the principle quantum number, crystal structure, chemical composition, and number of atoms.

\section{RESULTS AND DISCUSSION}

Figure 1.1 depicts the calculated absorption spectra as a function of wavelength of the incident photons for Ag nanoparticle of $4 \mathrm{~nm}$ in diameter containing 50 atoms. The 
figure shows only ten of all possible transitions from $5 \mathrm{~s}$ to 10s, 13s, 15s, 20s, 25s ( $n \geq 6, \Delta l=0)$ or to 10p, 13p, 15p, 20p, 25p $(n \geq 6, \Delta l=1)$. The absorption spectra are derived from the ground-state conduction electrons at the orbital 5s, which excite to higher conduction band energies at the orbital $n$ s or $n p$ or both with many possible transitions at $n \geq 6$ and $l=0$ or 1 . Each absorption spectrum represents one possible transition made by 50 conduction electrons and for each transition produces absorption peak $\lambda_{\max }$. It is interesting to note that despite many possible transitions, the discreteness of the conduction electron states does not clearly manifests itself because the absorption peak $\lambda_{\max }$ of each spectrum is very close to the absorption peaks of the other spectra.

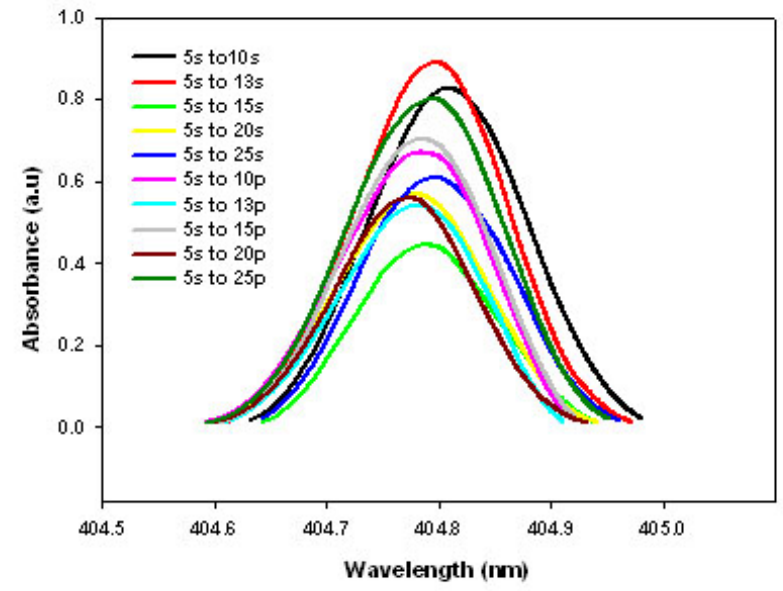

Figure 1.1: Calculated optical absorption spectra of 4-nm Ag nanosphere showing the absorption peaks of ten transitions from $5 \mathrm{~s}$ to $10 \mathrm{~s}, 13 \mathrm{~s}, 15 \mathrm{~s}$, 20s, 25s ( $n \geq 6, \Delta l=0$ ) and to 10p, 13p, 15p, 20p, 25p ( $n \geq 6$,

$\Delta l=1$ ). The energy bands of s-electrons and p-electrons in the conduction band are confined very close to each other and any possible transitions with quantum number $n \geq 6$ and $\Delta l=0$ or 1 produces the absorption peak revolves around $\lambda_{\max }=404.79 \mathrm{~nm}$ corresponds to the conduction band energy of $3.06 \mathrm{eV}$

We observe that the absorption peaks $\lambda_{\max }$ of the transitions shown appear in the narrow wavelength regimes between 404.77 and $404.81 \mathrm{~nm}$ for $4 \mathrm{~nm}$ Ag nanoparticle. This means that at higher energy regimes of the conduction band of s-electrons and p-electrons are confined very close to each other and can overlap. At a distance near to the Fermi level, which is extremely far from the centre of the particle, the nuclear potential of the conduction electrons is very small and any transition with allowed quantum numbers will have absorption energy equal to the conduction band energy. The final absorption spectrum of the Ag nanoparticle from all possible transitions is somewhat broadened but revolves about $\lambda_{\max }=404.79 \mathrm{~nm}$ corresponds to the conduction band energy of $3.06 \mathrm{eV}$.

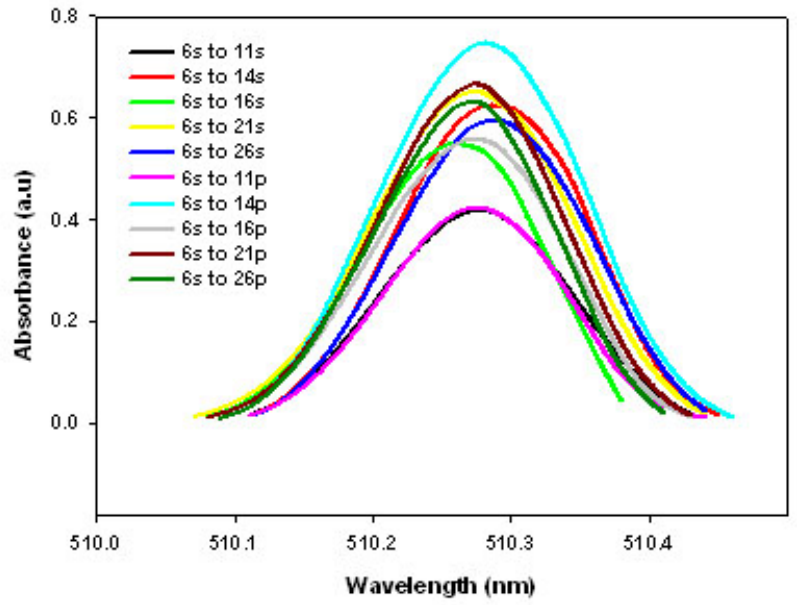

Figure 1.2: Calculated optical absorption spectra of 4-nm Au nanosphere showing the absorption peaks of ten transitions from $6 \mathrm{~s}$ to $11 \mathrm{~s}, 14 \mathrm{~s}, 16 \mathrm{~s}$, 21s, 26s ( $n \geq 7, \Delta l=0$ ) and to 11p, 14p, 16p, 21p, 26p ( $n \geq 7$, $\Delta l=1)$. The energy bands of s-electrons and p-electrons in the conduction band are confined very close to each other and any possible transitions with quantum number $n \geq 7$ and $\Delta l=0$ or 1 produces the absorption peak revolves around $\lambda_{\max }=510.28 \mathrm{~nm}$ corresponds to the conduction band energy of $2.43 \mathrm{eV}$.

Figure 1.2 depicts the calculated absorption spectra of ten possible transitions as a function of incident photon wavelength for a 4-nm $\mathrm{Au}$ nanoparticle containing 50 atoms. The spectra are derived from the ground-state conduction electrons $6 \mathrm{~s}$ to the orbital 11s, 14s, 16s, 21s, 26s $(n \geq 7, \Delta l=0)$ or to 11p, 14p, 16p, 21p, 26p ( $n \geq 7$, $\Delta l=1)$. Similar to the situation for Ag nanoparticle, the absorption peaks $\lambda_{\max }$ of Au nanoparticle appear in the narrow wavelength regime between 510.27 and $510.29 \mathrm{~nm}$. The final absorption spectrum of the Au nanoparticle should be a more broader spectrum owing to many possible transitions allowed by quantum number selection rules ( $n \geq 7$ and $\Delta l=0,1$ ). For the 4-nm Au nanoparticle the absorption peak revolves about $\lambda_{\max }=510.28 \mathrm{~nm}$ corresponds to the conduction band energy of $2.43 \mathrm{eV}$.

A red-shift of the absorption peak in metal nanoparticles has been well documented [34-36]. Figure 1.3, depicts the calculated absorption spectra of $\mathrm{Ag}$ nanoparticles at different sphere diameters of $4,5,7,10,15$, and $25 \mathrm{~nm}$, simulated for one of the possible transitions. We kept all parameters constant, except for the ground-state electron energy at $5 s$, which is dependent on the particle size or the number of atoms. The absorption peaks $\lambda_{\max }$ for Ag nanoparticles of diameters 4, 5, 7, 10, 15, and $25 \mathrm{~nm}$ appear at 404.79, 408.36, 412.55, 415.73, 418.42 and $420.96 \mathrm{~nm}$, respectively and are corresponding to the conduction band energy of 3.06, 3.04, 3.01, 2.98, 2.96, and $2.95 \mathrm{eV}$ respectively as shown in Table 1.1. 
The increase in absorption peak $\lambda_{\max }$ for different particle sizes is attributed to the increase in the number of atoms that make up the nanoparticles. The magnitude of the absorption peak $\lambda_{\max }$ shift is relatively substantial for the smaller particle sizes than that the larger sizes, indicating the absorption phenomenon of Ag nanoparticles is nonlinearly size dependence $[34,37,38]$. The wavelength $\lambda_{\max }$ red-shifts to higher wavelengths by increasing particle size and at the same time the spectrum becomes broaden with the size increases. Figure 1.4 depicts the calculated absorption spectra of Au nanoparticles at various diameters of $4,5,7,10,15$, and $25 \mathrm{~nm}$, which produce the absorption peaks at 510.28, 520.91, 533.11, 542.35, 549.74, and 556.04 $\mathrm{nm}$, respectively and are corresponding to the conduction band energy of 2.43, 2.37, 2.33, 2.29, 2.26, and 2.23 respectively as shown in Table 1.1 . We also observed that the absorption peaks $\lambda_{\max }$ nonlinearly red-shift to higher wavelengths with increasing Au nanoparticle sizes.

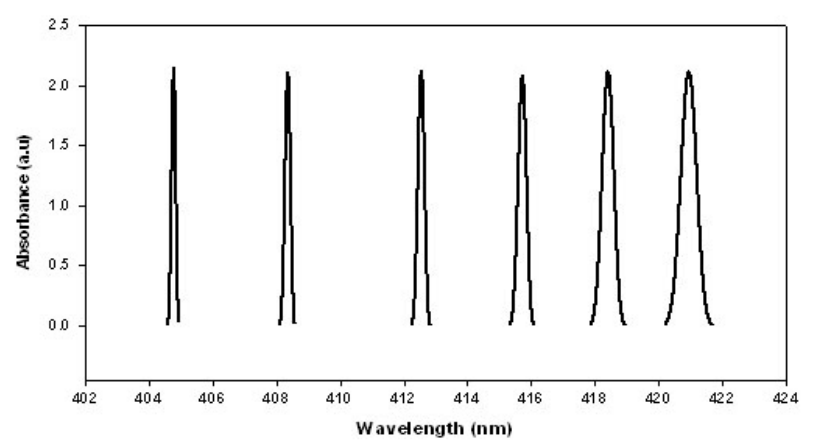

Figure 1.3: Calculated optical absorption spectra of Ag nanoparticles at various diameters of $4,5,7,10,15$, and $25 \mathrm{~nm}$, showing the absorption peaks $\lambda_{\max }$ appear at 404.78, 408.36, 412.55, 415.73, 418.42, and 420.96 $\mathrm{nm}$, respectively. The absorption peaks $\lambda_{\max }$ nonlinearly shift to higher wavelengths by increasing particle sizes and produce broader spectrum as the particle increases in size.

The appearance of the absorption peak $\lambda_{\text {max }}$ at distinct regimes between $\mathrm{Ag}$ and $\mathrm{Au}$ nanoparticles for a given nanosphere size is worth mentioning. The absorption peaks $\lambda_{\max }$ wavelengths of the 4-nm Ag and $\mathrm{Au}$ nanoparticles appear at $404.79 \mathrm{~nm}(3.06 \mathrm{eV})$ and $510.28 \mathrm{~nm}$ (2.43 eV), respectively, while for the 25-nm Ag and $\mathrm{Au}$ nanoparticles the peaks $\lambda_{\max }$ appear at $420.96 \mathrm{~nm}(2.95 \mathrm{eV})$ and $556.04 \mathrm{~nm}(2.23 \mathrm{eV})$ respectively. The discrepancy in the absorption peaks $\lambda_{\max }$ of the two nanoparticle systems is attributed to various factors, such as the multiplier parameter $\mu$, lattice constant $a$, atomic number $Z$, number of atoms $N$, and potential energy. The Fermi levels $\mu$ calculated for Ag and $\mathrm{Au}$ to be 5.49 and $5.53 \mathrm{eV}$, respectively. The Ag and Au nanoparticles are arranged in a face-centered cubic structure and their lattice constants $a$ calculated to be $0.408 \mathrm{~nm}$ and $0.407 \mathrm{~nm}$, respectively. We observed that the Fermi energy and lattice constant of Ag and Au are about equal, but the absorption peaks $\lambda_{\max }$ of the two systems are at different wavelength regimes. The reason is that the nuclear potential energy of the system is proportional to the atomic number $Z$ and inversely proportional to the displacement, $r$. The number of protons in $\mathrm{Ag}$ and $\mathrm{Au}$ atoms is 47 and 79, respectively. This produces a stronger potential energy to $\mathrm{Au}$ nanoparticles than Ag nanoparticles. Therefore, the conduction electrons of Au nanoparticles are attracted stronger towards the centre of the particle than Ag nanoparticles, reducing the size of the conduction band of $\mathrm{Au}$ nanoparticles more than that of Ag nanoparticles. Consequently, the absorption peak $\lambda_{\max }$ of Au nanoparticles is longer than the Ag nanoparticles.

Table 1.1: Calculated optical absorption wavelength $\lambda_{\max }$ and conduction band energy $E_{\mathrm{CB}}$ for Ag and Au nanoparticles of various sizes.

\begin{tabular}{|c|c|c|c|c|}
\hline \multirow{2}{*}{$\begin{array}{c}\text { Size } \\
(\mathbf{n m})\end{array}$} & $\begin{array}{c}\lambda_{\max } \\
\mathbf{( n m )}\end{array}$ & $\begin{array}{c}\boldsymbol{E}_{\mathrm{CB}} \\
\mathbf{( e V )}\end{array}$ & $\begin{array}{c}\lambda_{\max } \\
(\mathbf{n m})\end{array}$ & $\begin{array}{c}\boldsymbol{E}_{\mathrm{CB}} \\
(\mathbf{e V})\end{array}$ \\
\cline { 2 - 5 } & 404.79 & 3.06 & 510.28 & 2.43 \\
\hline 4 & 408.36 & 3.04 & 522.91 & 2.37 \\
\hline 5 & 412.55 & 3.01 & 533.11 & 2.33 \\
\hline 7 & 415.73 & 2.98 & 542.35 & 2.29 \\
\hline 10 & 418.42 & 2.96 & 549.74 & 2.26 \\
\hline 15 & 420.96 & 2.95 & 556.04 & 2.23 \\
\hline 25 & & & & \\
\hline
\end{tabular}

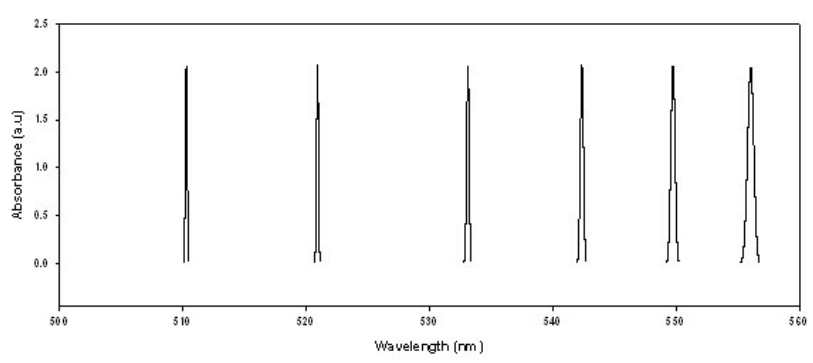

Figure 1.4: Calculated optical absorption spectra of Au nanoparticles at various diameters of $4,5,7,10,15$, and $25 \mathrm{~nm}$, showing the absorption peaks $\lambda_{\max }$ appear at 510.28, 522.91, 533.11, 542.35, 549.74, and 556.04 $\mathrm{nm}$, respectively. The absorption peaks $\lambda_{\max }$ nonlinearly shift to higher wavelengths by increasing particle sizes and produce broader spectrum as the particle increases in size.

\section{CONCLUSION}

In summary, we successfully used a quantum mechanical approach to calculate the optical absorption spectra of isolated Ag and Au nanoparticles based upon time-independent DFT. The absorption is derived from UVvisible photons impinging on the nanoparticles causing ground-state electrons of the conduction band excite to the 
higher energy states and the electronic transitions restricted only by the quantum number selection rules. The calculated absorption peaks $\lambda_{\max }$ of metal nanoparticles are sensitive to the particle type, which characterizes by the atomic number, Fermi energy, absorption, and potential energy of the system and on the particle size, which describes by the number of atoms and lattice constant. We have shown that the absorption peaks $\lambda_{\max }$ of the $\mathrm{Ag}$ and Au nanoparticles red-shift to the higher wavelengths by increasing particle size. The change in the wavelength shift is substantial at the smaller particle sizes than that the larger sizes, confirming the wavelength red-shift is nonlinearly size dependence. The quantum mechanical calculations of the absorption spectra, presented here for Ag and Au nanoparticles could be extended to other transition metal nanoparticles of interest in nanoscience and nanotechnology.

\section{ACKNOWLEDGEMENT}

This work was supported by the Ministry of Higher Education of Malaysia under the FRGS and RUGS grants.

\section{REFERENCES}

[1] W.A. Murray, J.R. Suckling, W.L. Barnes, Nano Lett., 6 (2006) 1772-1777.

[2] E. Ozbay, Science 331, (2006) 189-193.

[3] Y. Chen, K. Munechika, D.S. Ginger, Nano Lett, 7 (2007) 690-696.

[4] L.J. Sherry, S.H. Chang, G.C. Schatz, R.P. van Duyne , Nano Lett, 5 (2005) 2034-2038.

[5] N.K. Grady, N.J. Halas, P. Nordlander, Chem Phys Lett, 399 (2004) 167-171.

[6] C.J. Zhong, J. Luo, B. Fang, B.N. Wanjala, P.N. Njoki, R. Loukrakpam , J. Yin, Nanotechno, 21 (2010) 1-20.

[7] D.K. Kambhampati, W. Knoll, Curr. Opin. Colloid. In. Sci, 4 (1999) 273-280.

[8] K. Tanabe, Mater Lett, 61 (2007) 4573-4575.

[9] F. Cannone, M. Collini, L. D’Alfonso, G. Baldini, G. Chirico, G. Tallarida, P. Pallavicini, Nano Lett, 7 (2007) $1070-107$.

[10] G. Nikolai, N.G. Khlebtsov, L.A. Dykman, J Quant Spec Radiat Trans, 111 (2010) 1-35.

[11] G. Korotcenkov, S.D. Han , J.R. Stetter, Chem Rev, 109 (2009) 1402-1433.

[12] G. Mie, Annalen der Physik, 25 (1908) 377-445.

[13] M.A. Garcia, J. Llopis, S.E. Paje, Chem Phys Lett, 315 (1999) 313-320.

[14] V.M. Renteria, J. Garcia-Macedo, Colloids Surf. A, 273 (2006) 1-3.

[15] R. Guns, Ann Phys, 37 (1912) 881-900.

[16] J.C. Maxwell Garnett, Trans. R. Soc. London, 205 (1906) 237-288.

[17] A. Castro, M.A.L. Marques, J.A. Alonso, A.J. Rubio, Comp Theor Nanosci, 1 (2004) 231-255.

[18] D. Negrut, M. Anitescu, T. Munson, P. Zapol, in: J.M. Goicolea, J. Cuadrado, J.C. Garcia Orden (Eds), Density functional theory-based nanostructure investigation: Theoretical Considerations. Madrid, Spin, 21-24 June 2005 pp. 1-30.

[19] P. Samal P, M.K. Harbola, J Phys B: At Mol Opt Phys, 39 (2006) 4065-4080.

[20] C.M. Aikens, S. Li, G.C. Schatz, . J Phys Chem C, 112 (2008) 11272-11279.

[21] H. Chen, A. Zhou, Numer Math Theor Meth Appl, 1 (2008) 1-28.

J. Roqué, N. Poolton, J. Molera, A. Smith, E. Pantos, M. Vendrell-Saz, Phys Stat Solidi B, 243 (2006) 1337 - 1346

L.H. Thomas, Mathematical Proceedings of the Cambridge Philosophical Society 23 (1927) 542-548.

Hohenberg, W. Kohn, Phys Review, 136 (1964) B864-B871.

W. Kohn, L.J. Sham, Physical Review, 140 (1965) A1133-A1138.

E. Fermi, Rend Accad Naz Lincei, 6 (1927) 602-607.

P.A.M. Dirac, Mathematical Proceedings of the Cambridge Philosophical Society, 26 (1930) 376-385.

C.F. Von Weizsacker, Zeitschrift für Physik 96 (1935) 431-458.

W. Yang, Phys Review A, 34 (1986) 4575-4585.

P.K. Chattaraj, S. Sengupta, J Phys Chem A, 101 (1997) 7893-7900.

G.H. Chan , J. Zhao, E.M. Hicks, G.C. Schatz, R.P. Van Duyne, . Nano Lett, 7 (2007) 1947-1952.

N.W. Ashcroft, N.D. Mermin, Solid State Physics, W.B. Saunders Company, Philadelphia, 1976, pp. $135-138$.

W. Yang, R.G. Parr, C. Lee, Phys Rev, A34 (1986) 4586-4590.

A. Pan, Z. Yang , H. Zheng, F. Liu, Y. Zhu, X. Su, Z. Ding, Appl Surf Sci, 205 (1993) 323-328.

N. Sakai, Y. Fujiwara,M. Arai, K. Yu, T. Tatsuma, Electroanal Chem 628 (2009) 7-15.

Y. Peng, Y. Wang, Y. Yang, D.D. Dlott, J Nanopart Res, 12 (2009) 777-787.

B. Balamurugan, T. Maruyama, J Appl Phys, 102 (2007) 034306-034311.

[38] E. Anno, M. Tanimoto, Phy Rev B 73 (2006) 155430-155436. 